\title{
Mechanism of miR-98 inhibiting tumor proliferation and invasion by targeting IGF1R in diabetic patients combined with colon cancer
}

\author{
SHIXIONG LIU ${ }^{1}$, YUN ZHOU ${ }^{1}$, YONGNING ZHOU ${ }^{2}$, JING WANG ${ }^{1}$ and RUI JI ${ }^{2}$ \\ Departments of ${ }^{1}$ Geriatrics (II) and ${ }^{2}$ Gastroenterology, The First Hospital of Lanzhou University, \\ Lanzhou, Gansu 730000, P.R. China
}

Received November 15, 2019; Accepted December 03, 2019

DOI: $10.3892 / \mathrm{ol} .2020 .11707$

\begin{abstract}
Expression level of miR-98 in diabetic colon cancer (CRC) tissues and the regulation mechanism of colon cancer cell proliferation and invasion ability were studied. Forty patients with type 2 diabetes mellitus complicated with colon cancer, 40 colon cancer patients, and 40 patients with diabetic colonoscopy were enrolled between January 2017 and January 2018. Real-time quantitative PCR was used to detect the expression level of miR-98. After SW480 cells were transfected with miR-98 mimics or control simulants, the proliferation of cancer cells was detected by MTT assay, and the invasion ability of cancer cells was detected by Transwell cell invasion assay. The dual luciferase assay was used to detect the binding relationship between miR-98 and IGF1R. Western blot analysis was used to detect the expression of IGF1R protein in tumor tissues of patients with diabetes mellitus and colon cancer. Compared with diabetic patients, the expression level of miR-98 was decreased in colon cancer patients. Compared with tumor tissues of colon cancer patients, the expression level of miR-98 was significantly decreased in diabetic colon cancer tissues. Compared with the commonly cultured colon cancer SW480 cells, the expression level of miR-98 was significantly decreased in SW480 cells cultured under high glucose conditions. Increased expression of miR-98 inhibits colon cancer cell proliferation and invasion. miR-98 can target and bind to IGF1R and inhibit its expression level. IGF1R is upregulated in diabetic colon cancer tissue. miR-98 inhibits proliferation and invasion of diabetic colon cancer by targeting IGF1R. The expression level of miR-98 in diabetic colon cancer tissues is lower than that in colon cancer tissues. miR-98 can inhibit the proliferation and invasion of colon cancer cells by targeting
\end{abstract}

Correspondence to: Dr Shixiong Liu, Department of Geriatrics (II), The First Hospital of Lanzhou University, 1 Donggang West Road, Lanzhou, Gansu 730000, P.R. China

E-mail: shixiong_liu02@163.com

Key words: diabetes, colon cancer, miR-98, IGF1R, proliferation, invasion the target gene IGF1R. miR-98 may be a potential biological target for the treatment of patients with diabetes and colon cancer.

\section{Introduction}

Colon cancer is a common gastrointestinal cancer. More than 900,000 patients are diagnosed with colon cancer each year, and its incidence is the third among all cancers (1). Colon cancer is ranked third of cancer-related deaths (2). In most colon cancer patients, the spread of tumor cells is the main cause of death of patients. Most patients with stage I and II colon cancer can be cured by surgical resection. Approximately $70 \%$ of patients with colon cancer with stage III lymph node metastasis can be cured by surgery combined with adjuvant chemotherapy. It is difficult to cure advanced metastatic colon cancer (stage IV) (3). Despite significant advances in surgical techniques and adjuvant chemotherapy, the survival rate of colon cancer patients has only modest improvement due to the high recurrence rate of colon cancer and advanced colon cancer at the time of diagnosis $(2,4)$. Therefore, the pathogenesis of colorectal cancer needs to be further explored.

Studies have shown that diabetes, especially type 2 diabetes, can increase the risk of cancer and death, especially in gastrointestinal cancers (5). Type 2 diabetes is an independent risk factor for colon cancer, and colon cancer patients with diabetes have a worse prognosis than those without diabetes (6). In addition, in diabetic patients, the incidence of colon cancer increased by 1.27-1.40 times (7). Moreover, patients with colon cancer and type 2 diabetes had a 5-year survival rate reduction of $42 \%$ and a $21 \%$ increase in colon cancer recurrence (8). However, the reasons for this increase in risk are unclear. Some scholars have suggested that the increase in the level of free insulin growth factor 1 (IGF1) may promote the proliferation of colon cancer cells $(9,10)$. However, the specific regulatory mechanisms are still unclear.

In recent years, most human genome transcripts have been transcribed into non-coding RNAs, such as small non-coding RNAs (miRNAs). miRNAs do not encode any protein, but can regulate biological processes by inhibiting the expression level of target gene mRNA, thereby regulating the occurrence and progression of the disease (11-13). Studies have shown that 
miRNAs are differentially expressed in a variety of cancers, and differentially expressed miRNAs can be involved in the development and progression of cancer (14-17), including colon cancer (18-20). For example, the expression level of miR-3653 is significantly downregulated in colon cancer tissues and cells, and the increased expression level of miR-3653 can significantly inhibit the migration and invasion of colon cancer cells by inhibiting the expression level of target gene Zeb2. It also inhibits the epithelial-mesenchymal transition (ERT) of colon cancer cells (18). miR-223-3p, which is significantly upregulated in colon cancer tissues, can promote colon cancer cell proliferation, invasion and migration by inhibiting the target gene PRDM1 expression level (19). Among miRNAs, miR-98 is differentially expressed in a variety of cancers and can regulate a variety of cancer cell phenotypes. For example, miR-98, which is downregulated in breast cancer tissues and cell lines, can further regulate proliferation, invasion and migration ability of breast cancer cells by targeting the expression level of the target gene Gab2 (GRB2-associated-binding protein 2) (21). miR-98 is downregulated in the expression of squamous cell carcinoma of the head and neck can also regulate the proliferation and invasion of cancer cells (22). In addition, studies have found that miR-98 expression levels are downregulated in colon cancer and can be used as tumor suppressors to regulate the Warburg effect of colon cancer cells (23). However, the current regulatory role and mechanism of miR-98 in patients with diabetes mellitus complicated with colon cancer is not clear. Therefore, in this experiment, we mainly explored the differential expression of miR-98 and its mechanism of regulation in colon cancer combined with type 2 diabetes mellitus.

\section{Materials and methods}

Tissue sample collection and cell culture. Tumor tissues of 40 patients with type 2 diabetes mellitus complicated with colon cancer and 40 colon cancer patients were collected between January 2017 and January 2018. Of the 40 patients with type 2 diabetes mellitus and colon cancer, 21 were male and 21 were female, aged of $61.7 \pm 5.9$ years. Of the 40 colon cancer patients, 22 were male and 18 were female, aged $63.1 \pm 6.5$ years. Diagnostic standard for type 2 diabetes was based on 1999 World Health Organization (WHO) criteria for the diagnosis of type 2 diabetes: i) patients with a history of significant diabetes; ii) oral glucose tolerance test $2 \mathrm{~h}$ blood glucose $\geq 11.1 \mathrm{mmol} / \mathrm{l}$. Patients with other types of diabetes and those with a history of hyperthyroidism and glucocorticoid use were excluded. Colon cancer patients were diagnosed by histopathology and did not receive any treatment. Patients with diabetes mellitus combined with colon cancer and colon cancer patients were in stage T3-T4 of colon cancer. Informed consent was obtained from all the patients. The experimental protocol was approved by the Ethics committee of The First Hospital of Lanzhou University.

The human colon cancer cell line SW480 from ATCC (American Type Culture Collection) was purchased from Beijing Zhongyuan Heju Economic and Trading Co., Ltd. SW480 was cultured in DMEM medium supplemented with $10 \%$ fetal bovine serum, and $100 \mathrm{U} / \mathrm{ml}$ penicillin and
$100 \mu \mathrm{g} / \mathrm{ml}$ streptomycin were added to prevent cell contamination. SW480 cells were cultured at $37^{\circ} \mathrm{C}$, in $5 \% \mathrm{CO}_{2}$ cell culture incubator. SW480 cells were divided into two groups, one group was cultured using conventional medium, and the other group was cultured using medium supplemented with $25 \mathrm{mM}$ glucose $(24,25)$.

Cell transfection. The human colon cancer SW480 cells in logarithmic growth phase were prepared into cell suspensions, and the cells were inoculated in 6-well plates at about $6 \times 10^{5}$ cells per well. The 6 -well plates were placed at $37^{\circ} \mathrm{C}$ culture chamber with $5 \% \mathrm{CO}_{2}$ continued for $24 \mathrm{~h}$. The control mimic, miR-98 mimic (miR-98 mimic) or miR-98 mimic and IGF1R overexpression plasmid (pCDNA-IGF1R) were then transfected into cells alone using the lipofection reagent Lipofectamine 3000. After $72 \mathrm{~h}$ of transfection, the transfected SW480 cells were collected for subsequent experiments.

Real-time QPCR. Total RNA in tumor tissues of diabetic colon cancer patients, adjacent normal tissues, colon cancer SW480 cells, and human normal colon epithelial cells NCM460 were extracted using TRIzol reagent. Using the extracted RNA as a template, a reverse transcription reaction was carried out using a reverse transcription kit (Revert Aid RT Reverse Transcription kit, K1691, Thermo Fisher Scientific, Inc.) to obtain cDNA. Then, cDNA was used as a template, and the expression level of miR-98 was detected by PCR reaction using a SYBR Green Master Mix kit (4385618, Thermo Fisher Scientific, Inc.) in the Mx3000P Real-Time PCR system, and U6 was used as an internal reference. The miR-98 primer sequence was: upstream primer, 5'-GGACTGAGGTAGTAAGTTG-3'; downstream primer, 5'-CATCAGATGCGTTGCGTA-3' (26). The U6 primer sequence was: upstream primer, 5'-GCTTCGGCA GCACATATACTAAAAT-3'; downstream primer, 5'-CGC TTCACGAATTTGCGTGTCAT-3'. The PCR reaction procedure was: 1 cycle of $\left(9^{\circ} \mathrm{C}, 5 \mathrm{~min}\right), 42$ cycles of $\left(92^{\circ} \mathrm{C}, 15 \mathrm{sec}\right.$, $\left.60^{\circ} \mathrm{C}, 15 \mathrm{sec}, 72^{\circ} \mathrm{C}, 30 \mathrm{sec}\right)$. The relative expression levels of miR-98 were analyzed using the $2^{-\Delta \Delta \mathrm{Ct}}$ method.

MTT experiment. SW480 cells in the logarithmic growth phase were made into cell suspensions and seeded into 96-well plates at a seeding density of $5 \times 10^{3}$ cells per well, and then placed at $37^{\circ} \mathrm{C}$, in $5 \% \mathrm{CO}_{2}$ cell culture incubator and cultured for 3 days. After the completion of the culture, $20 \mu \mathrm{l}$ of a $5 \mathrm{mg} / \mathrm{ml}$ MTT reagent was added to each well of a 96-well plate, and then the 96-well plate was further cultured in a cell culture incubator containing $5 \% \mathrm{CO}_{2}$ at $37^{\circ} \mathrm{C}$ for $4 \mathrm{~h}$. After $4 \mathrm{~h}, 150 \mu \mathrm{l}$ of the reagent was added to each well of a 96-well plate to dissolve the crystals. Finally, the absorbance was measured at a wavelength of $570 \mathrm{~nm}$.

Transwell cell invasion assay. The upper chamber of the Transwell chamber for cell invasion experiments was first uniformly coated with $50 \mu 1$ of Matrigel, and DMEM containing $10 \%$ fetal calf serum was added to the lower chamber of the Transwell chamber. Then, a well-grown SW480 cell suspension was inoculated into the upper chamber of the Transwell chamber, and the inoculated cell density was $1 \times 10^{5}$. SW480 cells in the upper chamber were placed at 

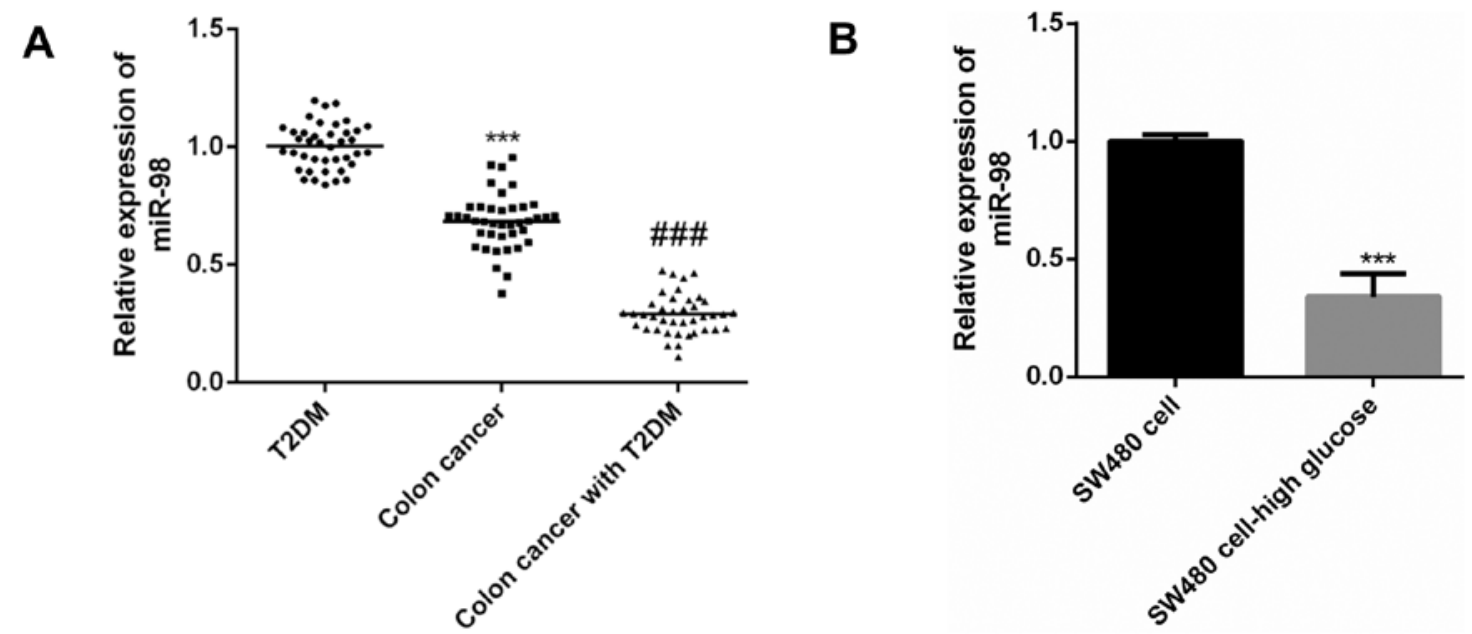

Figure 1. Expression levels of miR-98 in diabetic colon cancer patients and colon cancer cell lines. (A) Real-time quantitative PCR technology detection of the expression level of miR-98 in diabetic patients, colon cancer patients and tumor tissues of patients with diabetes and colon cancer. (B) Real-time quantitative PCR detection of the difference in expression levels of miR-98 between colon cancer cell line SW480 and SW480 cells cultured under high glucose conditions. ${ }^{* * *} \mathrm{P}<0.001,{ }^{\# \# \#} \mathrm{P}<0.001$.

$37^{\circ} \mathrm{C}$, in $5 \% \mathrm{CO}_{2}$ cell incubator and cultured with serum-free medium for $24 \mathrm{~h}$. After the completion of the culture, SW480 cells on the surface of the membrane were washed, and the remaining SW480 cells were fixed with $4 \%$ paraformaldehyde and stained with $0.1 \%$ crystal violet for $10 \mathrm{~min}$. Finally, five regions were randomly selected from the microscope field to count the invasive SW480 cells.

Bioinformatics methods to screen miR-98 target genes. In order to find potential target genes for miR-98, bioinformatics software miRWalk database (http://www.ma.uni-heidelberg. de/apps/zmf/mirwalk/) miRanda (http://www.microrna. org/microrna/getExprForm.do), miRDB (http://mirdb. org/), miRNAMAP (http://mirnamap.mbc.nctu.edu.tw/) and TargetScan (http//www.targetscan.org) were used for analysis. Screening principle: miR-98 was complementary binding to the target gene locus; miR-98 target gene locus did not contain a complex secondary structure, binding site was highly conserved, miR-98 and target gene mRNA were highly thermostable. According to the predicted results, the IGF1R gene may be a target gene of miR-98.

Dual luciferase assay. The target gene of miR-98 was verified using a dual luciferase assay. The IGF1R gene wild-type 3'-untranslated region (3'-UTR) and mutant 3'-UTR were constructed into the pmiRGLO vector (Promega). The vector carrying the wild-type 3'-UTR and mutant 3'-UTR of the IGF1R gene was then transfected into SW480 cells using the lipofection reagent Lipofectamine 3000, respectively, and then the wild-type 3'-UTR SW480 cells were divided into two subgroups, one subgroup transfected the miR-98 mimetic, and the other subgroup transfected the control mimic, and the mutant 3'-UTR SW480 cells were also divided into two subgroups, one subgroup transfected with miR-98 mimics, another subgroup was transfected with control mimics. After $48 \mathrm{~h}$ of transfection, the relative luciferase activity in colon cancer cells was measured using a microplate reader.
Western blot analysis. Total protein in colon cancer SW480 cells was extracted using RIPA lysate. The total protein concentration after extraction was quantified using a BCA protein quantification kit. Lysate $(40 \mu \mathrm{g})$ was separated using $12 \%$ SDS-PAGE and transferred to a PVDF membrane. The PVDF membrane was blocked with $4 \%$ skim milk powder for $1 \mathrm{~h}$, and the mouse anti-human IGF1R protein antibody (ab16890, Abcam) (dilution, 1:500) or mouse anti-human GAPDH protein antibody (ab8245, Abcam) (dilution, 1:500) was incubated overnight at $4^{\circ} \mathrm{C}$ with PVDF membrane, then incubated with HRP-linked rabbit anti-mouse IgG secondary antibody (ab6728, Abcam) (dilution, 1:2000) under $37^{\circ} \mathrm{C}$ condition. In this experiment, GAPDH protein was used as an internal reference protein. Finally, color development was performed using an ECL chemiluminescence chromogenic kit, and imaging was performed using a ChemiDoc MP chemiluminescence imaging system. Western blot bands were quantified using ImageJ software.

Statistical analysis. Statistical analysis was performed using SPSS 22.0, and the data was expressed using mean \pm standard deviation. Differences between the two groups were analyzed using an independent sample t-test. $\mathrm{P}<0.05$ was considered a statistically significant difference.

\section{Results}

miR-98 is downregulated in tumor tissues and colon cancer cell lines of patients with diabetes mellitus complicated with colon cancer. According to the results of real-time PCR, the expression level of miR-98 in colon cancer patients was significantly lower than that in diabetic patients $(\mathrm{P}<0.001)$, compared with colon cancer patients, the expression level of miR-98 in patients with diabetes and colon cancer was significantly decreased $(\mathrm{P}<0.001)$ (Fig. 1A). Compared with human colon cancer SW480 cells cultured under normal conditions, miR-98 was also significantly decreased in colon cancer cell line SW480 cultured under high glucose conditions $(\mathrm{P}<0.01)$ 
A

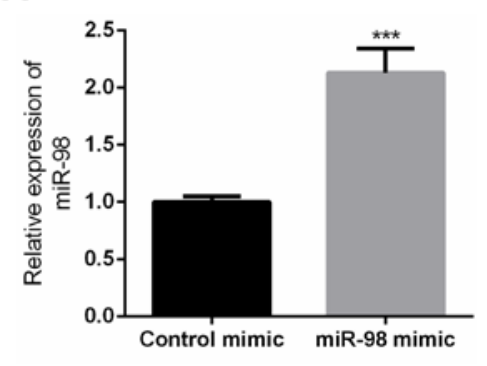

C

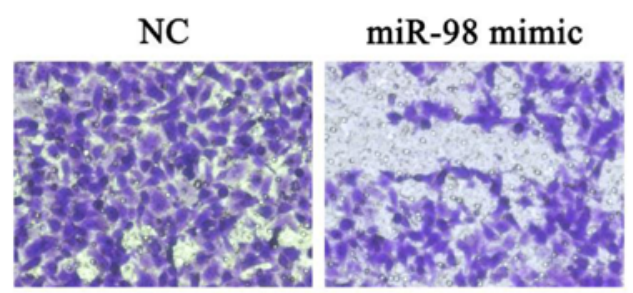

B

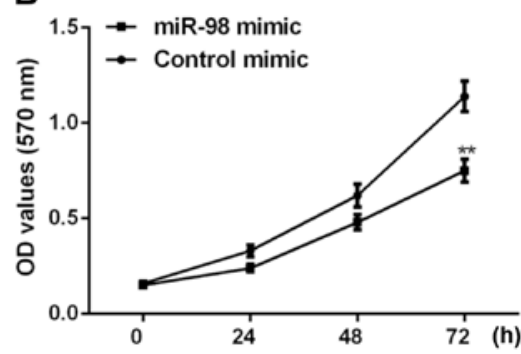

D

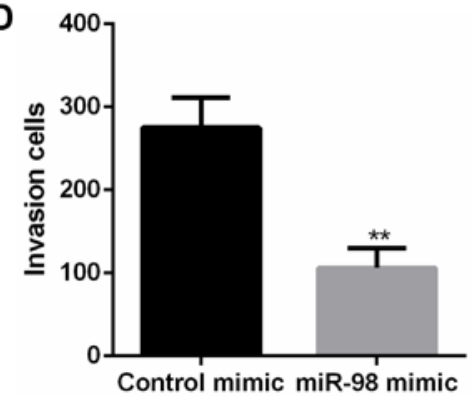

Figure 2. Effect of upregulation of miR-98 expression on proliferation and invasion of colon cancer cells. (A) Real-time quantitative PCR was used to detect the expression level of miR-98 in SW480 cells transfected with miR-98 mimic. (B) MTT assay was used to detect the effect of upregulation of miR-98 expression on the proliferation of colon cancer cells. (C) Transwell cell invasion assay was used in upregulation of miR-98 expression on the invasion ability of colon cancer cells. (D) Statistical results of invasion of colon cancer cells after upregulation of miR-98. ${ }^{* *} \mathrm{P}<0.01,{ }^{* * *} \mathrm{P}<0.001$.

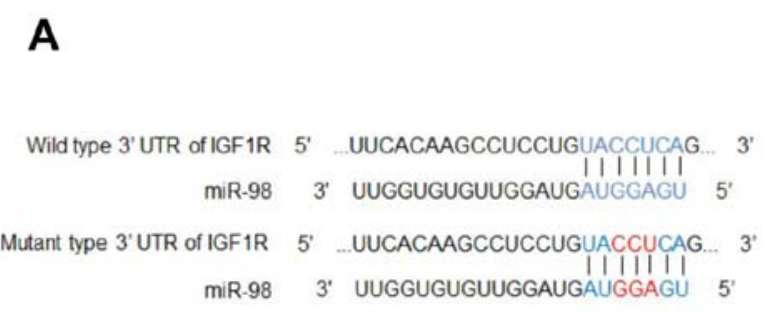

C

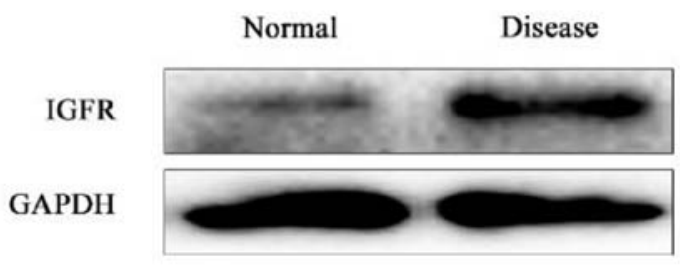

B

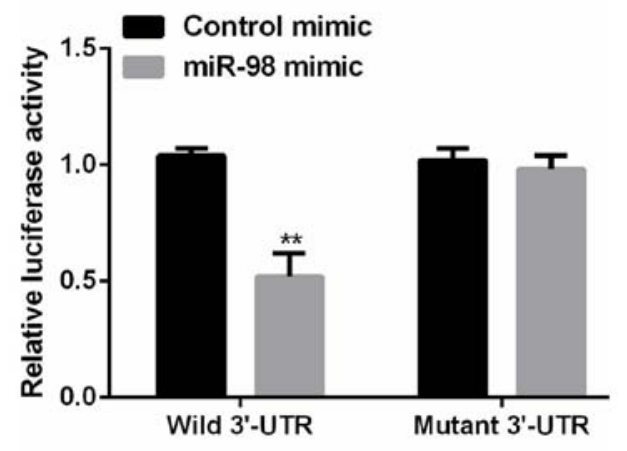

D

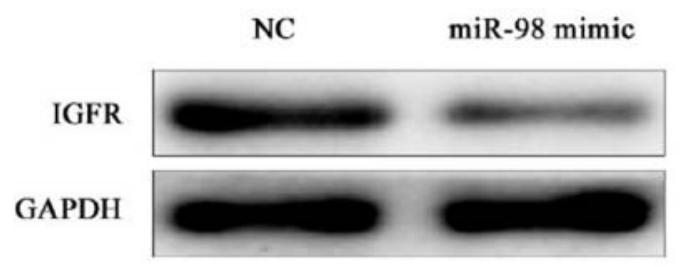

Figure 3. Targeting the relationship between miR-98 and IGF1R and its effect on IGF1R expression levels. (A) Sequence of potential binding sites for miR-98 and IGF1R. (B) Dual luciferase assay detection of the relationship between miR-98 and IGF1R. (C) Western blot analysis of the expression level of IGF1R in tumor tissues and adjacent normal tissues of patients with diabetes and colon cancer. (D) Western blot analysis of the effect of upregulation of miR-98 expression on IGF1R expression levels. ${ }^{* *} \mathrm{P}<0.01$.

(Fig. 1B). Therefore, miR-98 has a decreased expression level in tumor tissues of diabetic patients with colon cancer and colon cancer cell lines under high glucose conditions.

Upregulation of miR-98 expression inhibits colon cancer cell proliferation and invasion. After transfecting control mimic and miR-98 mimic into SW480 cells, real-time PCR was performed. According to the results, miR-98 mimic can upregulate the expression level of miR-98 in SW48 cells compared with SW480 cells transfected with control mimic $(\mathrm{P}<0.001)$ (Fig. 2A). After the expression level of miR-98 was upregulated, the proliferation of SW480 cells was detected. 

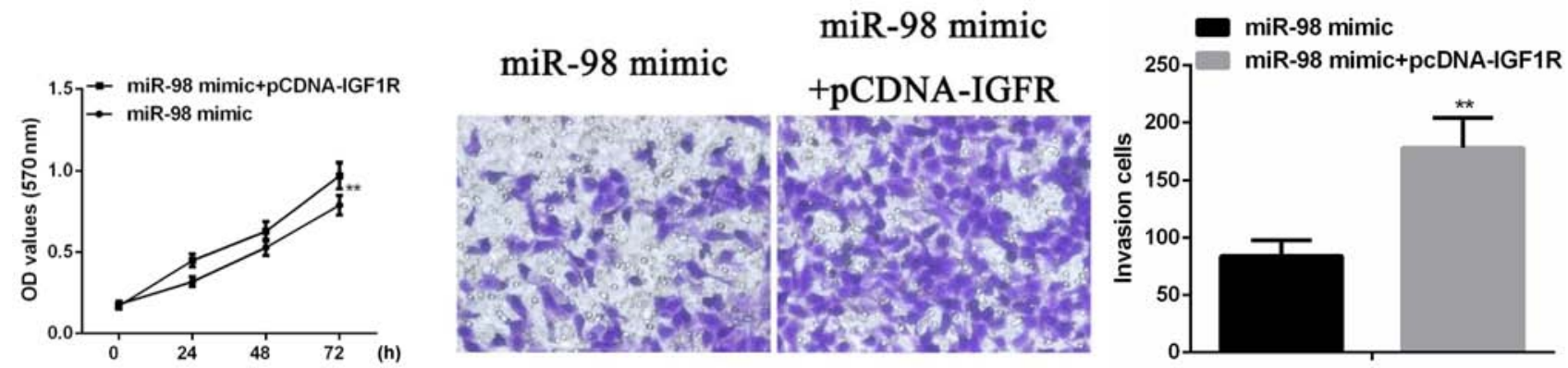

Figure 4. miR-98 regulates proliferation and invasion of colon cancer cells by regulating the target gene IGF1R. (A) MTT assay was used to detect the effect of miR-98 and IGF1R expression levels on the proliferation of colon cancer cells. (B) Transwell cell invasion assay was used to detect the effect of miR-98 and IGF1R expression levels on the invasion ability of colon cancer cells. ${ }^{* *} \mathrm{P}<0.01$

According to the results of MTT assay, the upregulation of miR-98 significantly inhibited cell proliferation compared with the control mimic group $(\mathrm{P}<0.01)$ (Fig. 2B). Transwell cell invasion assay showed that the invasive ability of SW480 cells was significantly decreased $(\mathrm{P}<0.01)$ after upregulation of miR-98 expression compared with control mimic group (Fig. 2C). According to the above results, the upregulation of miR-98 expression can inhibit the proliferation and invasion of colon cancer cells.

miR-98 targets inhibition of its target gene IGF1R expression level. It is predicted by the bioinformatics database that IGF1R may be a target gene of miR-98, and the potential binding sequence of miR-98 and IGF1R 3'-UTR is shown in Fig. 3A. Dual luciferase assay was performed to further verify whether there is a targeted binding relationship between miR-98 and IGF1R. The results showed that there was no significant difference in relative luciferase activity between the $\mathrm{miR}-98 \mathrm{mimic}$ group and the control group in the SW480 cells transfected with the IGF1R mutant 3'-UTR, contrary to the SW480 cells transfected with the IGF1R wild-type 3'-UTR. Compared with the control group, miR-98 mimic transfection significantly reduced the relative luciferase activity in SW480 cells $(\mathrm{P}<0.01)$ (Fig. 3B). Western blot analysis showed that the protein expression level of IGF1R was significantly higher in tumor tissues of patients with diabetes mellitus complicated with colon cancer than in adjacent normal tissues (Fig. 3C). In addition, IGF1R protein expression levels were significantly downregulated after upregulation of miR-98 expression levels compared to controls (Fig. 3D). Based on the above results, miR-98 can target and bind to the target gene IGF1R and negatively regulates its expression level.

miR-98 regulates proliferation and invasion of colon cancer cells by regulating target gene IGF1R. To further investigate the regulation of miR-98 on the behavior of colon cancer cells, miR-98 mimic and pCDNA-IGF1R were simultaneously transfected into SW480 cells, and cell proliferation and invasion ability were examined. According to the results of MTT assay, simultaneous transfection of miR-98 mimic and pCDNA-IGF1R significantly increased the proliferation of colon cancer cells compared with SW480 cells transfected with miR-98 mimic alone $(\mathrm{P}<0.01)$ (Fig. 4A). Transwell cell invasion assay showed that transfection of miR-98 mimic and pCDNA-IGF1R significantly increased the invasiveness of colon cancer cells compared with SW480 cells transfected with miR-98 mimic alone $(\mathrm{P}<0.01)$ (Fig. 4B).

\section{Discussion}

In order to clarify the pathogenic mechanism of patients with diabetes mellitus complicated with colon cancer, miR-98 was used as the research object in this study. Firstly, the expression level of miR-98 in patients with diabetes mellitus and colon cancer was studied, and then the influence of the change of miR-98 expression level on the proliferative capacity and invasive ability of human colon cancer cells under high glucose conditions was studied, followed by bioinformatics analysis and dual luciferase assay to identify potential target genes of miR-98, and the effects of the target genes on the proliferation and invasion of human colon cancer cells under high glucose conditions were explored, in order to clarify the regulatory mechanism of miR-98 on diabetes and colon cancer, and to explore potential biological targets for the treatment of diabetic colon cancer.

First, in order to study whether there is a difference in the expression level of miR-98 in tumor tissues of patients with diabetes mellitus complicated with colon cancer and tumor tissues of colon cancer patients, 40 tumor patients with diabetes mellitus combined with colon cancer and 40 colon cancer patients after surgery, 40 colon cancer biopsies from patients with diabetes mellitus were collected. and the expression of miR-98 in colon cancer patients was lower than that in diabetic patients by real-time fluorescent quantitative PCR. However, the expression level of miR-98 in tumor tissues of diabetic patients combined with colon cancer was also significant lower than that of colon cancer patients, and the expression level of miR-98 in colon cancer cell lines cultured under high glucose conditions was found to be significantly lower than the colon cancer cell line cultured under normal conditions. According to literature reports, the expression level of miR-98 in tumor tissues was significantly reduced. For example, the expression level of miR-98 was significantly decreased in tumor tissues of patients with non-small cell lung cancer (27). In laryngeal squamous cell carcinoma tumor tissues and laryngeal squamous cell carcinoma cell lines, the 
expression level of miR-98 was also significantly lower than that of normal tissues and normal cell lines (28). In retinoblastoma, miR-98 expression levels were also downregulated (29). In breast cancer patients' tumor tissues and breast cancer cell lines, the expression level of miR-98 was also found to be significantly lower than that of adjacent normal tissues and normal cell lines (21). In addition, a team found that miR-98 was expressed at low levels in tumor tissues and colon cancer cells of colon cancer patients (23). In this experiment, the difference in the expression level of miR-98 between colon cancer cell lines and normal cells was not detected, but miR-98 was found to have lower expression level under high glucose conditions. The expression level of miR-98 in diabetic colon cancer patients was lower than colon cancer patients, suggesting that miR-98 may have a regulatory role in diabetes with colon cancer.

The differential expression levels of miRNAs in diseased and normal tissues usually indicate that miRNAs may play important regulatory roles in disease. For example, miR-98, which is significantly downregulated in tumor tissues of non-small cell lung cancer patients and non-small cell lung cancer cell lines, can regulate the migration and invasion ability of non-small cell lung cancer cells (27). miR-98 is downregulated in tumor tissues and laryngeal squamous cell carcinoma cell lines of laryngeal squamous cell carcinoma, its upregulation can inhibit the ERT of tumor cells and the invasive behavior of laryngeal squamous cell carcinoma cell lines (28). The expression level of miR-98 is downregulated in retinoblastoma, its upregulation can inhibit the proliferation, invasion and migration ability of retinoblastoma cells (29). In breast cancer, upregulation of miR-98 expression by cell transfection can inhibit breast cancer cell proliferation, invasion, migration, and epithelial to mesenchymal transition (21). Therefore, we hypothesized that the significant decrease in the expression level of miR-98 in diabetic and colon cancer tissues in this experiment may indicate that miR-98 can also regulate the behavior of cancer cells in this disease. Therefore, we performed MTT assay and Transwell cell invasion assay to investigate the changes in proliferation and invasion ability of colon cancer cells after upregulation of miR-98 expression. According to the experimental results, it was found that after the colon cancer cell line transfected with miR-98 mimic significantly upregulated the expression level of miR-98, the cell proliferation and invasion ability of colon cancer cell lines were significantly inhibited. This result is similar to the regulation of miR-98 on cancer cell behavior in other cancers. At the same time, the results of this experiment are similar to those found by other scholars in miR-98 in colon cancer. The researchers found that miR-98, which is significantly downregulated in tumor tissues and colon cancer cell lines of colon cancer patients, after significantly increasing the expression level, it can significantly inhibit the proliferation of colon cells. In addition, they also found that upregulation in miR-98 expression level can inhibit glucose absorption and lactate secretion, that is, inhibit the glycolysis process of colon cancer cells. Glycolysis process is the main functional metabolism of cancer cells. If the glycolysis process is inhibited, it can inhibit the proliferation of cancer cells (23). In this experiment, we did not investigate the effect of miR-98 on the metabolic patterns of colon cancer cells.
miRNAs are a class of non-coding RNAs that do not encode any protein, but can be involved in the regulation of gene expression levels at the post-translational level. The most important way for miRNAs to regulate gene expression levels is through targeting the non-translated region of mRNA to mediate the post-translational silencing function of the gene, and the key region of the sequence complementarity of the miRNA to its target is located in the seed region of the 2-7 nucleotides at the 5' end of the miRNA (30). In cancer, the main regulatory effect of miRNAs on cancer cell proliferation, invasion and migration ability is through the regulation of the expression level of its target genes. For example, in lung cancer cell lines, miR-335 can target and negatively regulate the expression level of transformer 2 beta homolog (Tra2 $\beta$ ), and then regulate the proliferation of lung cancer cells (31). In gastric cancer, miR-129-5p and miR-129-3p can regulate the proliferation and migration ability of gastric cancer cells by regulating the expression level of target gene WWP1 (32). Moreover, miR-98 has also been found to regulate the cellular behavior of cancer cells by regulating the expression levels of target genes. For example, miR-98 can inhibit the cell proliferation, invasion and migration ability of non-small cell lung cancer by targeting inhibition of TWIST expression levels (27). Furthermore, miR-98e can also inhibit the progression of retinoblastoma by targeting HMGA2 (29). In breast cancer, miR-98 can inhibit the proliferation and metastasis of breast cancer cells by targeting to inhibit the expression level of Gab2. In this experiment, we found that miR-98 can inhibit the proliferation and invasion of colon cancer cells, thus, we speculate that miR-98 may inhibit the proliferation and invasive ability of colon cancer cells by targeting the expression of target genes in this experiment. Therefore, we first screened the potential target gene of miR-98 through bioinformatics software, and then carried out the dual luciferase assay to further verify the target gene of miR-98. The experimental results showed that IGF1R is a target gene of miR-98. It can be targeted to bind to the 3'-UTR region by miR-98. Moreover, western blot analysis showed that the expression level of IGF1R protein was significantly up-regulated in tumor tissues of diabetic patients with colon cancer, which was contrary to the expression of miR-98 in tumor tissues of patients with diabetes mellitus with colon cancer. Furthermore, we found that IGF1R protein expression levels were significantly inhibited when miR-98 expression levels were up-regulated. Based on the above results, it is suggested that miR-98 can target to bind IGF1R, and miR-98 can negatively regulate the expression level of IGF1R protein. According to literature, we speculate that IGF1R protein may mediate the regulation of miR-98 on the proliferation and invasion of colon cancer cells. Therefore, we also changed the expression levels of miR-98 and IGF1R by cell transfection, and found that up-regulation of IGF1R expression can reverse the inhibition of colon cancer cell proliferation and invasion ability caused by up-regulation of miR-98 expression. Therefore, it is known that the inhibitory effect of miR-98 on the proliferation and invasion ability of colon cancer cells is achieved by targeted binding to IGF1R.

The insulin-like growth factor receptor IGF1R is a receptor protein located on the cell surface and binds to insulin-like growth factor. This receptor binds to a ligand to transmit 
cellular signals, causing cell growth and division. IGF1R is found to be highly expressed in tumor cells, which promotes the proliferation of cancer cells (33), and IGF1R is considered to be a potential biological target for cancer therapy, similar to the results of this experiment. In addition, in the study of diabetes mellitus complicated with colon cancer, some scholars have suggested that the increase in the level of free insulin growth factor may promote the proliferation of colon cancer cells $(9,10)$. We hypothesized that IGF1R may have a regulatory role in this process. In diabetic colon cancer cells, decreased expression of miR-98 leads to increased expression of IGF1R, elevated levels of IGF1R binds to more insulin growth factors, transmitting cellular signals, leads to cancer cell proliferation and malignant progression of the tumor. Therefore, based on the results of the present study, we consider that miR-98 may be a potential biological target for the treatment of patients with diabetes and colon cancer.

In conclusion, the expression level of miR-98 is downregulated in diabetic colon cancer tumor tissues, and the proliferation and invasion ability of colon cancer cells are inhibited by targeted inhibition of the expression level of the target gene IGF1R. miR-98 may be a potential biological target for the treatment of patients with diabetes mellitus combined with colon cancer.

\section{Acknowledgements}

Not applicable.

\section{Funding}

No funding was received.

\section{Availability of data and materials}

The datasets used and/or analyzed during the current study are available from the corresponding author on reasonable request.

\section{Authors' contributions}

SL put forward the proposition, designed the study and wrote the manuscript. YuZ, performed PCR, MTT and Transwell assay. YoZ was responsible for Dual luciferase assay and western blot analysis. JW and RJ contributed to the analysis of the observation indexes. The final version was read and adopted by all the authors.

\section{Ethics approval and consent to participate}

The study was approved by the Ethics Committee of The First Hospital of Lanzhou University (Lanzhou, China). Informed consent was obtained from all the patients.

\section{Patient cnsent for publication}

Not applicable.

\section{Competing interests}

The authors declare that they have no competing interests.

\section{References}

1. Peng YC, Lin CL, Hsu WY, Chang CS, Yeh HZ, Liao SC and $\mathrm{Kao} \mathrm{CH}$ : The risk of colorectal cancer is related to frequent hospitalization of IBD in an Asian population: results from a nationwide study. QJM 108: 457-463, 2015.

2. Belov L, Zhou J and Christopherson RI: Colorectal cancer therapeutic antibodies. In: Encyclopedia of Cancer. Schwab M (ed). Springer, Berlin, Heidelberg, pp944-948, 2014.

3. Ling H, Pickard K, Ivan C, Isella C, Ikuo M, Mitter R, Spizzo R, Bullock M, Braicu C, Pileczki V, et al: The clinical and biological significance of miR-224 expression in colorectal cancer metastasis. Gut 65: 977-989, 2016.

4. Dienstmann R, Vermeulen L, Guinney J, Kopetz S, Tejpar S and Tabernero J: Consensus molecular subtypes and the evolution of precision medicine in colorectal cancer. Nat Rev Cancer 17: 79-92, 2017.

5. Al Omari A, Abdelkhaleq H, Al-Hussaini M, Turfa R, Awad N, Hassan MM, Alfaqih MA and Garrett CR: Validation of the survival benefits of metformin in middle Eastern patients with type II diabetes mellitus and colorectal cancer. J Glob Oncol 4: 1-10, 2018.

6. Park JW, Lee JH, Park YH, Park SJ, Cheon JH, Kim WH and Kim TI: Sex-dependent difference in the effect of metformin on colorectal cancer-specific mortality of diabetic colorectal cancer patients. World J Gastroenterol 23: 5196-5205, 2017.

7. Nakayama Y, Iijima T, Wakaume R, Takahashi K, Matsumoto H, Nakano D, Miyaki M and Yamaguchi T: Microsatellite instability is inversely associated with type 2 diabetes mellitus in colorectal cancer. PLoS One 14: e0215513, 2019.

8. Meyerhardt JA, Catalano PJ, Haller DG, Mayer RJ, Macdonald JS, Benson AB III and Fuchs CS: Impact of diabetes mellitus on outcomes in patients with colon cancer. J Clin Oncol 21: 433-440, 2003.

9. Berster JM and Göke B: Type 2 diabetes mellitus as risk factor for colorectal cancer. Arch Physiol Biochem 114: 84-98, 2008.

10. Jin T: Why diabetes patients are more prone to the development of colon cancer? Med Hypotheses 71: 241-244, 2008.

11. Wu YZ, Chan KYY, Leung KT, Lam HS, Tam YH, Lee KH, Li K and Ng PC: Dysregulation of miR-431 and target gene FOXA1 in intestinal tissues of infants with necrotizing enterocolitis. FASEB J 33: 5143-5152, 2019.

12. Shen B, Han S, Wang Y, Yang Z, Zou Z, Liu J, Zhao Z, Wu R and Wang C: Bta-miR-152 affects intracellular triglyceride content by targeting the UCP3 gene. J Anim Physiol Anim Nutr (Berl) 103: 1365-1373, 2019

13. Zhang X, Zhang M, Guo Q, Hu X, Zhao Z, Ni L, Liu L, Wang X, Wang Z, Tong D, et al: MicroRNA-1297 inhibits proliferation and promotes apoptosis in gastric cancer cells by downregulating CDC6 expression. Anticancer Drugs 30: 803-811, 2019.

14. Ghaemi Z, Soltani BM and Mowla SJ: MicroRNA-326 functions as a tumor suppressor in breast cancer by targeting ErbB/PI3K signaling pathway. Front Oncol 9: 653, 2019.

15. Yun Z, Meng F, Jiang P, Yue M and Li S: microRNA-548b suppresses aggressive phenotypes of hepatocellular carcinoma by directly targeting high-mobility group box $1 \mathrm{mRNA}$. Cancer Manag Res 11: 5821-5834, 2019.

16. Liu J, Huang Y, Cheng Q, Wang J, Zuo J, Liang Y and Yuan G: miR-1-3p suppresses the epithelial-mesenchymal transition property in renal cell cancer by downregulating Fibronectin 1. Cancer Manag Res 11: 5573-5587, 2019.

17. Wang X, An D, Liu X, Wang X and Li B: MicroRNA-27a downregulates the expression of Hsp90 and enhances the radiosensitivity in esophageal squamous cell carcinoma. OncoTargets Ther 12: 5967-5977, 2019.

18. Zhu W, Luo X, Fu H, Liu L, Sun P and Wang Z: miR-3653 inhibits the metastasis and epithelial-mesenchymal transition of colon cancer by targeting Zeb2. Pathol Res Pract 215: 152577, 2019.

19. Chai B, Guo Y, Cui X, Liu J, Suo Y, Dou Z and Li N: miR-223-3p promotes the proliferation, invasion and migration of colon cancer cells by negative regulating PRDM1. Am J Transl Res 11: 4516-4523, 2019.

20. Jin Y, Cheng H, Cao J and Shen W: MicroRNA 32 promotes cell proliferation, migration, and suppresses apoptosis in colon cancer cells by targeting OTU domain containing 3. J Cell Biochem 120: 18629-18639, 2019.

21. Shi XY, Wang H, Wang W and Gu YH: miR-98-5p regulates proliferation and metastasis of MCF-7 breast cancer cells by targeting Gab2. Eur Rev Med Pharmacol Sci 23: 2847-2855, 2019. 
22. Tan H, Zhu G, She L, Wei M, Wang Y, Pi L, Chen C, Zhang D, Tan P, Chen J, et al: miR-98 inhibits malignant progression via targeting MTDH in squamous cell carcinoma of the head and neck. Am J Cancer Res 7: 2554-2565, 2017.

23. Zhu W, Huang Y, Pan Q, Xiang P, Xie N and Yu H: MicroRNA-98 suppress Warburg effect by targeting HK2 in colon cancer cells. Dig Dis Sci 62: 660-668, 2017.

24. Del Puerto-Nevado L, Minguez P, Corton M, Solanes-Casado S, Prieto I, Mas S, Sanz AB, Gonzalez-Alonso P, Villaverde C, Portal-Nuñez S, et al; Diabetes Cancer Connect Consortium: Molecular evidence of field cancerization initiated by diabetes in colon cancer patients. Mol Oncol 13: 857-872, 2019.

25. Wang L, Bai YY, Yang Y, Hu F, Wang Y, Yu Z, Cheng Z and Zhou J: Diabetes mellitus stimulates pancreatic cancer growth and epithelial-mesenchymal transition-mediated metastasis via a p38 MAPK pathway. Oncotarget 7: 38539-38550, 2016.

26. Wang $\mathrm{K}$, Dong L, Fang Q, Xia $\mathrm{H}$ and Hou X: Low serum miR-98 as an unfavorable prognostic biomarker in patients with non-small cell lung cancer. Cancer Biomark 20: 283-288, 2017.

27. Zhou H, Huang Z, Chen X and Chen S: miR-98 inhibits expression of TWIST to prevent progression of non-small cell lung cancers. Biomed Pharmacother 89: 1453-1461, 2017.

28. Zhu M, Zhang C, Chen D, Chen $S$ and Zheng $H$ : MicroRNA-98-HMGA2-POSTN signal pathway reverses epithelial-to-mesenchymal transition in laryngeal squamous cell carcinoma. Biomed Pharmacother 117: 108998, 2019.
29. Li W, Wang J, Zhang D, Zhang X, Xu J and Zhao L: MicroRNA-98 targets HMGA2 to inhibit the development of retinoblastoma through mediating Wnt/ $\beta$-catenin pathway. Cancer Biomark 25: 79-88, 2019.

30. Acunzo M, Romano G, Wernicke D and Croce CM: MicroRNA and cancer - a brief overview. Adv Biol Regul 57: 1-9, 2015.

31. Liu J, Bian T, Feng J, Qian L, Zhang J, Jiang D, Zhang Q, Li X, Liu Y and Shi J: miR-335 inhibited cell proliferation of lung cancer cells by target Tra2 $\beta$. Cancer Sci 109: 289-296, 2018.

32. Ma L, Chen X, Li C, Cheng R, Gao Z, Meng X, Sun C, Liang C and Liu Y: miR-129-5p and -3p co-target WWP1 to suppress gastric cancer proliferation and migration. J Cell Biochem: Nov 11, 2018. (Epub ahead of print). doi: 10.1002/jcb.28027.

33. Kumar AS, Rayala SK and Venkatraman G: Targeting IGF1R pathway in cancer with microRNAs: How close are we? RNA Biol 15: 320-326, 2018.

This work is licensed under a Creative Commons Attribution-NonCommercial-NoDerivatives 4.0 International (CC BY-NC-ND 4.0) License. 